\title{
A PRELIMINARY BOTANICAL SURVEY OF THE BIG MUDDY VALLEY IN SOUTHERN SASKATCHEWAN, 1968
}

\section{by Helen Morrison, Regina}

\section{Introduction}

The Big Muddy Valley is located in south central Saskatchewar, approximately 100 miles south and slightly west of Regina. The valley begins south of the town of Harptree in Township 4, Range 26, West of the 2nd Meridian, where Twelve Mile Lake Valley and Lake of the Rivers Valley meet, and extends southeast to Big Muddy Lake. South from the lake, a branch of the valley, often referred tuas the lower Big Muddy Valley, continues south across the International Boundary to join the Missouri Valley. With its steep walls of exposed bedrock and interesting rock outcrop formations, the Big Muddy Valley is a sharp contrast to the undulating prairie through which it cuts.

To the best of my knowledge, no intensive study of the flora of the Big Muddy Valley has previously been made. The herbarium of the Saskatchewan Museum of Natural History in Regina contains a number of specimens collected in the Big Muddy Valley by William Shevkenek (1937, 1938, and 1939). Some collections were also made in this area for the Museum by O. C. Furniss (1949), L. T. Carmichael (1949), and Dr. G. F. Ledingham (1950). The herbarium at the University of Saskatchewan, Regina Campus, has specimens collected from the Big Muddy Valley in recent years by Mr. and Mrs. D. Wade and by Dr. G. F. Ledingham.

Under the guidance of Dr. G. F. Ledingham, I chose part of the Big Muddy region as a study area (Map 1). From May to October, 1968, this study area was visited at about twoweek intervals, weather permitting. Sixteen collection sites in various habitats in the area were designated

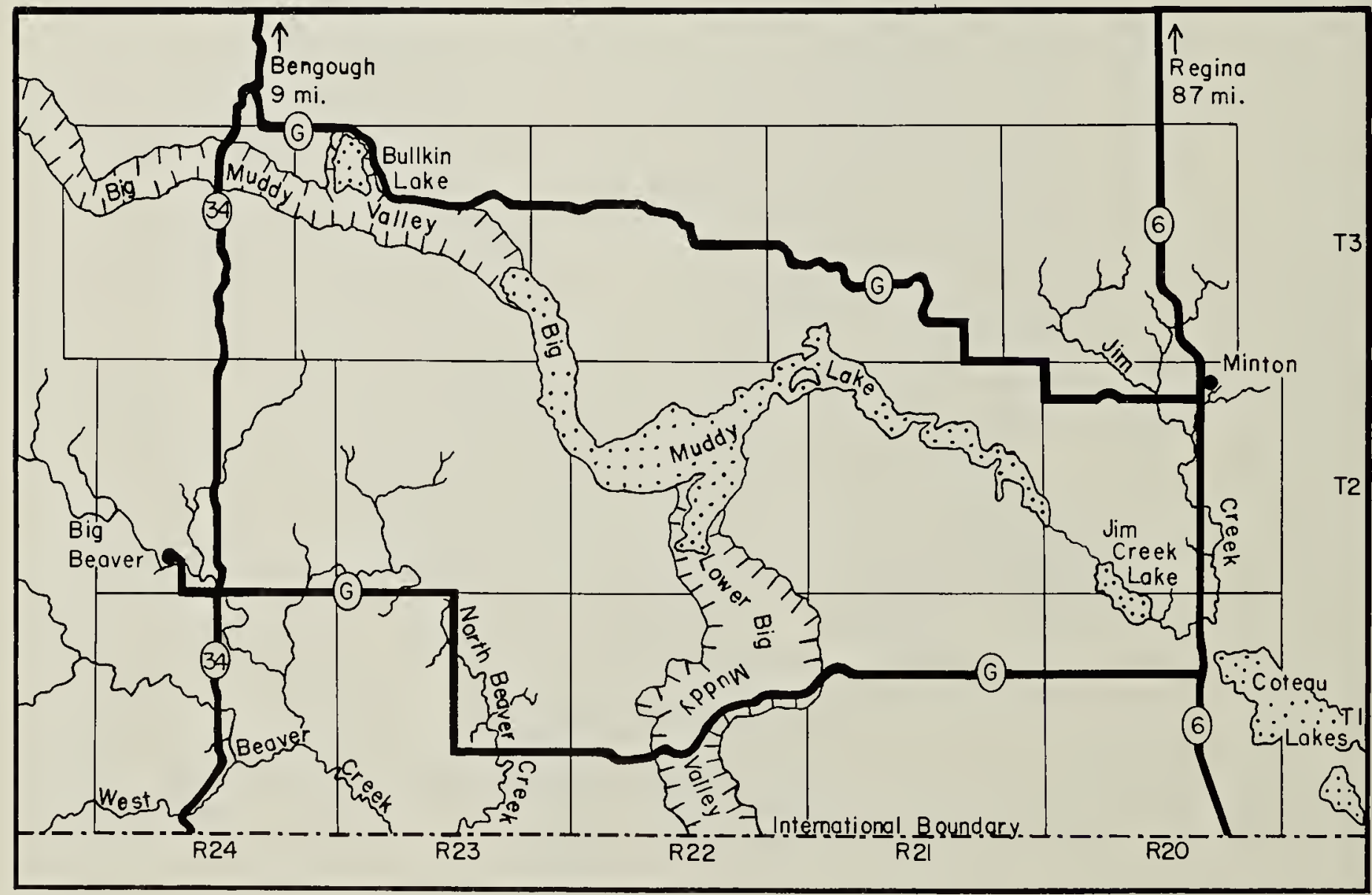

Map 1. The general location of the Big Muddy study area, its main roads and general geographical features. 


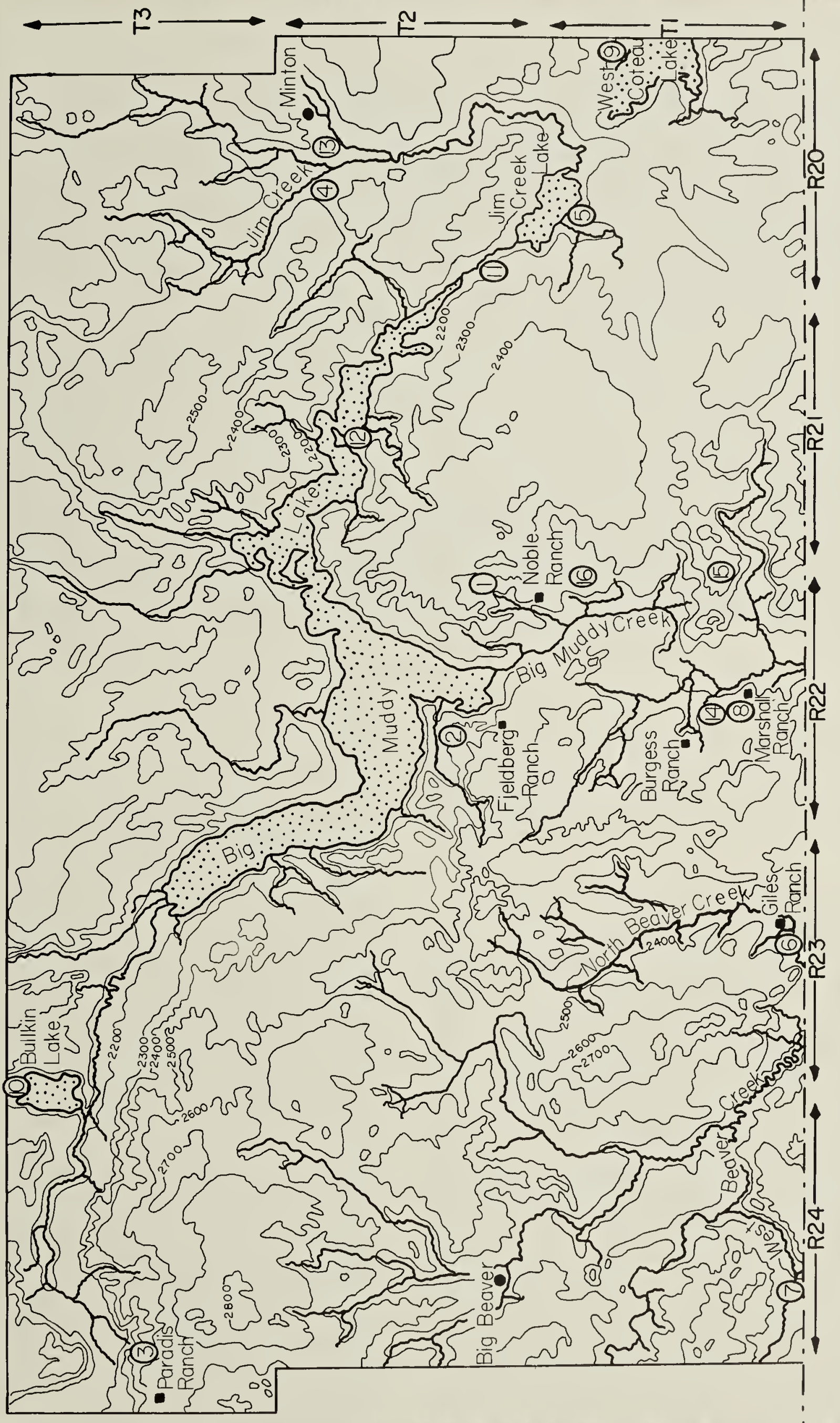

Tap 2. Topographical features of the Big Muddy study area and the location the main collection sites. Contour interval is 100 feet. 
and visited regularly (Map 2). Collections were made in other areas when time allowed. During this period, 336 species were collected. The list of species which follows is by no means a complete list of vascular plants for the area, but it is a preliminary list to which additions can be made in the future. As this was the first botanical work of this nature that I have attempted, there is no doubt that some species were overlooked in the field. The pattern of precipitation during the months of the study is another factor which probably influenced the number of species found. Because the months from May to July were exceptionally dry, it is possible that many perenniais did not flower. The total precipitation recorded at Minton for these three months was 2.94 inches. In August and September, the recorded precipitation at Minton was 7.23 inches and 1.16 inches respectively*. It was not unusual to see early-flowering species such as the Crocus (Anemone patens var. wolfgangiana) and Threeflowered Avens (Geum triflorum) blooming in late September. Perhaps these plants were in a state of dormancy earlier due to insufficient moisture.

\section{Study Area}

\section{A. Description}

The area chosen for study, Townships 1-3, Ranges 20-24, West of 2 nd Meridian, consists of 540 square miles including the eastern end of the main Big Muddy Valley, Big Muddy Lake, and the lower Big Muddy Valley. This area, located mainly between No. 6 and No. 34 highways, is crossed by two grid roads, one north and one south of Big Muddy Lake, making the area easily accessible by car (Map 1).

The study area lies completely within the semi-arid brown soil zone. According to Soil Map of Saskatchewan, Map 7, Soil Survey Report No. 10 , the valley is characterized by badlands with eroding clay soils (Figure

\footnotetext{
* Monthly Record of Meteorological Observations in $\mathrm{Canada}$, Regina Weather Office.
}

1), while the surrounding uplands, fo the most part, are covered by Have hill Association loam, rated poorly a a grain producer, with some areas Haverhill Association clay loam. Th floor of the main Big Muddy Valle and the flat extending from the ea end of Big Muddy Lake to We Coteau Lake are classified as heav alkali soils. Although most sloughs the area are alkaline, there are man fresh springs which run throughou the year.

The present drainage system in th valley had no part in the valley formation. Creeks and runoffs lead Big Muddy Lake from north, wes and south. In the east, Jim Creek w? dammed in 1952 to form Jim Cree Lake. West Coteau Lake is extremel alkaline, and, like Big Muddy Lake contained very little water in 196 until the heavy rains in August. Th north and west forks of Beaver Cree have their sources southwest of $\mathrm{Bi}$ Muddy Lake but flow south across th International Boundary to the Mis souri River System. Bullkin Lak northwest of Big Muddy, also lie within the study area.

A variety of habitats were found i this region - alkaline flats, marsh creeks and springs, eroding clay bad lands with rock outcrops, undulatin and rolling prairie uplands, mois wooded coulees and depressions, an disturbed areas. By designating collec tion localities in the different habitat and visiting them regularly, I attempt ed to collect as many species as pos sible. Sites 1 to 3 were located in th badlands, sites 4 to 8 were marsh areas near creeks and springs, sites to 13 were on extremely alkaline soil: site 14 was a rock outcrop, site 1 covered various wooded derpression: and site 16 was grazed natural pas ture land. Specimens from disturbe areas were collected mainly fror roadsides throughout the entire stud area.

\section{B. Geology}

The Big Muddy Valley was forme approximately 15,000 years ago dus ing the retreat of the final ice shee 


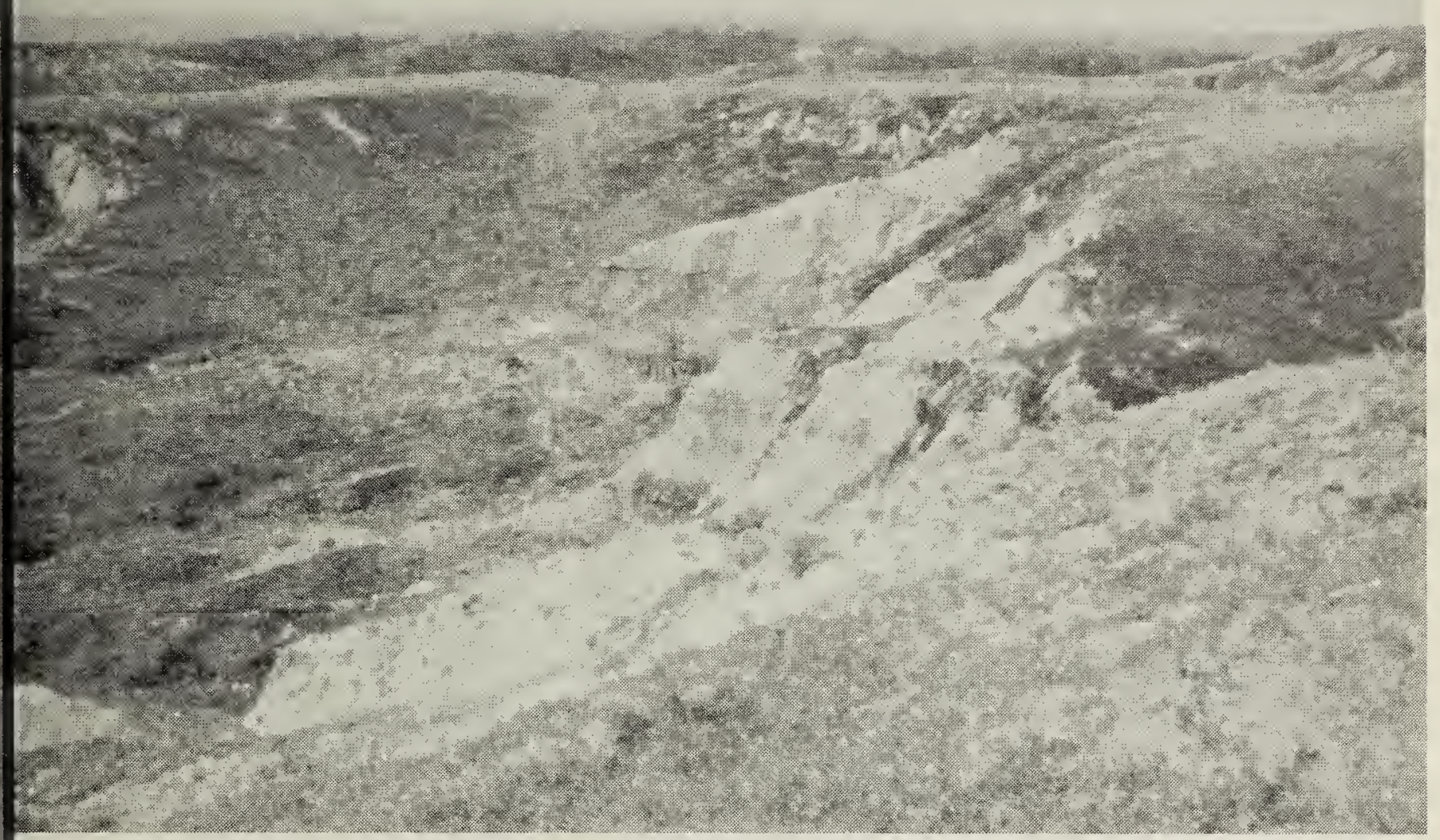

ig. 1. Big Coulee on the Noble ranch in the lower valley showing eroding ay slopes characteristic of the badlands.

com southern Saskatchewan. As the e retreated in a northeast direction, eltwater from the glacier front cut is channel in the bedrock and later andoned it. The valley has since equired a level floor of alluvium, cared down by runoff water in wet years. ow the valley averages 250 feet in epth, although south of Big Muddy ake, the depth reaches 500 feet Houldsworth, 1941). Slumping and eathering have made the steep slopes ore gentle until today many are rassy, but bare strata can be seen in any places along the length of the alley.

The lowest of the bedrock strata en in the part of the Big Muddy alley located in the study area is the hitemud formation, seen to the reatest extent where No. 34 highway cosses the valley. Here an uplift has curred, causing a thickness of 50 et to become visible. Two miles east the west end of Big Muddy Lake, is stratum dips below the present ke level, and it is not visible in the wer Big Muddy Valley. A white hd light grey sandy clay zone forms the lower part of this formation, while the upper zone is of white, grey, pale mauve, and black carbonaceous clay containing silt beds (Parizek, 1964).

The Upper Ravenscrag formation, which may be as thick as 500 feet, rests on the Whitemud formation as the Lower Ravenscrag is generally missing in this area. It contains many coal seams. The lower part of this zone, the Grey Facies, is made up of feldspathic sand, often cross-bedded, and grey, greenish-grey, and yellow sandy clay beds while the upper Buff Facies are composed of yellowishbrown fine-grained sand, silt, shale, and clay (Parizek, 1964). The exposures in the lower Big Muddy Valley also exhibit the Willowbunch clay meander.

The vast quantities of clays and sands of these formations were laid down long before glaciation by river systems which brought the material from highlands in the west, after the expulsion of the seas from western Canada. 


\section{Fauna}

During trips in the field, I saw various faunal species. I will attempt at this time to give an account only of the vertebrate animals I observed while in the study area.

Pronghorns, generally in small groups, were a common sight in the lower Big Muddy Valley and north of Big Muddy Lake. W. Noble, whose ranch is in the lower valley, reported that large herds of Pronghorns often feed with his cattle during winters when the snow is deep. Coyotes were noted on many occasions throughout the study area. The Mule Deer is also common in this part of the province. Smaller mammals seen included Nuttall': Cottontail, White-tailed Jack Rabbit, Red Fox, Richardson's Ground Squirrel, Thirteen-lined Ground Squirrel, and the Striped Skunk. On one field trip to the area Big Brown Bats were collected from crevices in rocks on the south shore of Big Muddy Lake (by Gary Anweiler for U. of S., Regina Campus, September, 1968).

An outstanding feature of the bird population of the Big Muddy area is the numerous species of hawks found there. Those species present are the Ferruginous Hawk, Sharp-shinned Hawk, Cooper's Hawk, Marsh Hawk, Red-tailed Hawk, Swainson's Hawk, Pigeon Hawk, and Sparrow Hawk; the Prairie Falcon also nests in the valley. (Bernie Haysom, personal communication). Two Golden Eagle nests were located in the survey area.

Several kinds of snakes appear to be common in the lower valley including the Plains Garter Snake and Bull Snake. The Smooth Green Snake is present in the area also (collected by Dr. D. Secoy of U. of S., Regina Campus, September, 1968 Cf. Blue. Jay, 26:p. 203). An Eastern Yellowbellied Racer was killed by T. Marshall on his ranch in the lower Big Muddy in September, 1968.

\section{Flora of Big Muddy Valley}

The grasses found in this region are characteristic of the Mixed Prairie. Little Bluestem (Andropogan scoparius), Blue Grama Grass (Bouteloua gracilis), Spear Grass (Stipa vir dula), and June Grass (Koeleria cris tata) were very plentiful. The exter sive growth in pastures of species suc as Prairie Selaginella (Selaginell densa), Pasture Sage (Artemisi frigida), Broomwe ed (Gutierrezi sarothrae), Prickly P e a r Cactu (Opuntia polyacantha), and Cushio Cactus (Mamillaria vivipara) ind cated the problem of overgrazin faced by ranchers. The Lands Branc of Saskatchewan Department of Agr culture indicates that, depending o land management procedures, in thi part of the province from 20 to 3 acres of native pasture is required pe head of cattle, and, to replenish pas tures, $40 \%$ of the grasses should b allowed to go to seed annually.

The dominant grass on alkalin flats in this region was Alkali Gras (Distichlis stricta). Various member of the family Chenopodiaceae wer prevalent also, including Greasewoo (Sarcobatus vermiculatus), Wester Sea-blite (Suaeda depressa), and Sam phire (Salicornia rubra). Small-leave Everlasting (Antennaria parvifoli Nutt.) was common in alkaline area also.

Various species of sedges (Care spp.), bulrushes (Scirpus spp.), an rushes (Juncus spp.) were associate with creeks and springs in the $\mathrm{Bi}$ Muddy area (Figure 2). Seasid Arrow-grass (Triglochin maritima Seaside Buttercup (Ranunculus cym balaria), and Salt-meadow Gras (Puccinellia nuttalliana) were ver abundant in these areas, and Willow (Salix spp.) were found occasionall around sloughs.

Vegetation cover is relatively scar in the "badlands". Many of the stee slopes were bare, while the mor gentle slopes had some cover (Figur 3). Creeping Juniper (Juniperus hor zontalis) and Low Juniper (J.con munis) formed large mats over th clay slopes, preventing further dow ward erosion (Figure 3 and 4). Ral bit bush (Chrysothamnus nauseosus and Shrubby Cinquefoil (Potentil, fruticosa) were widespread here. Ye 


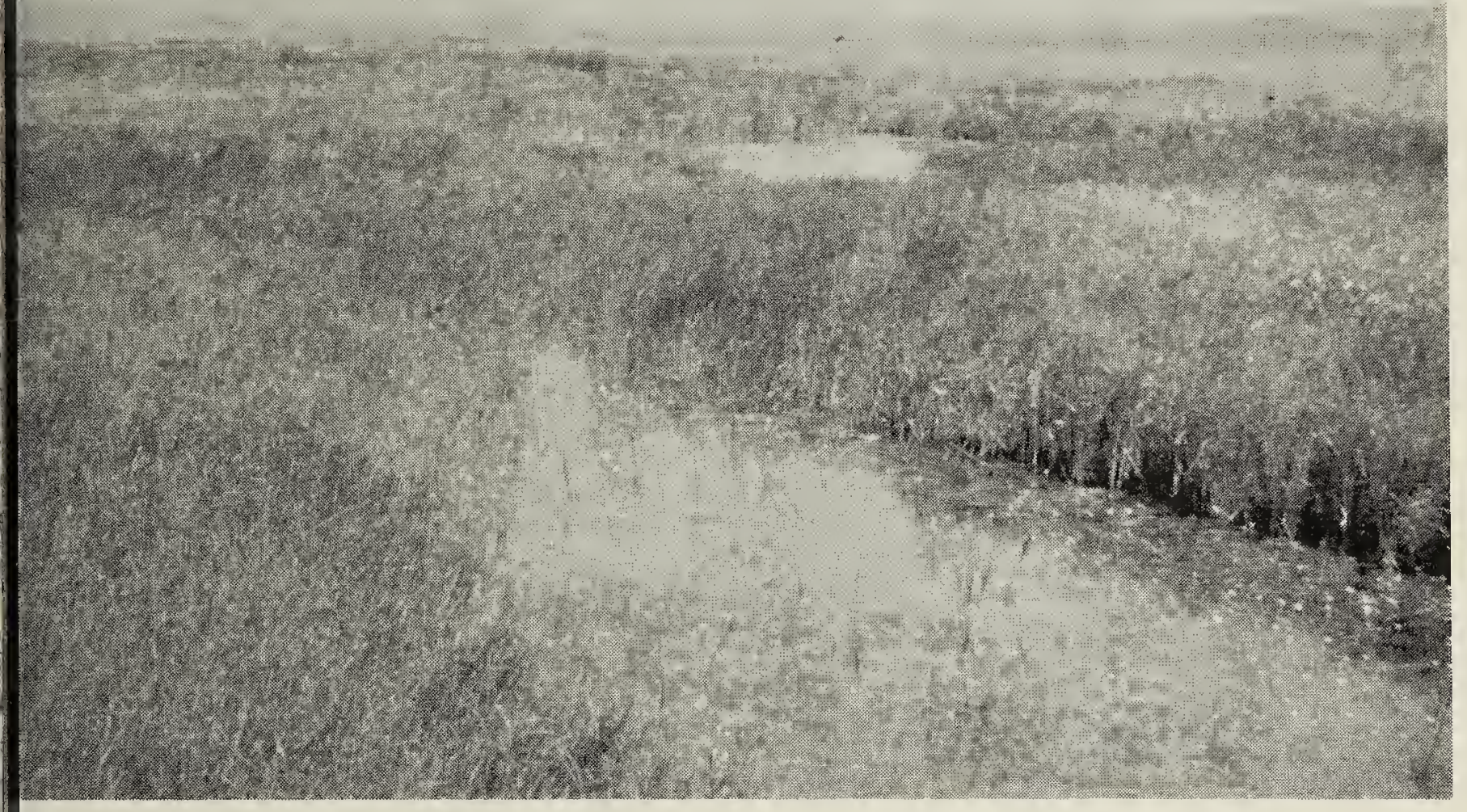

ig. 2. Jim Creek, one mile west of Minton, looking north from the grid road, lowing thick growth of Scirpus, Carex and Juncus.

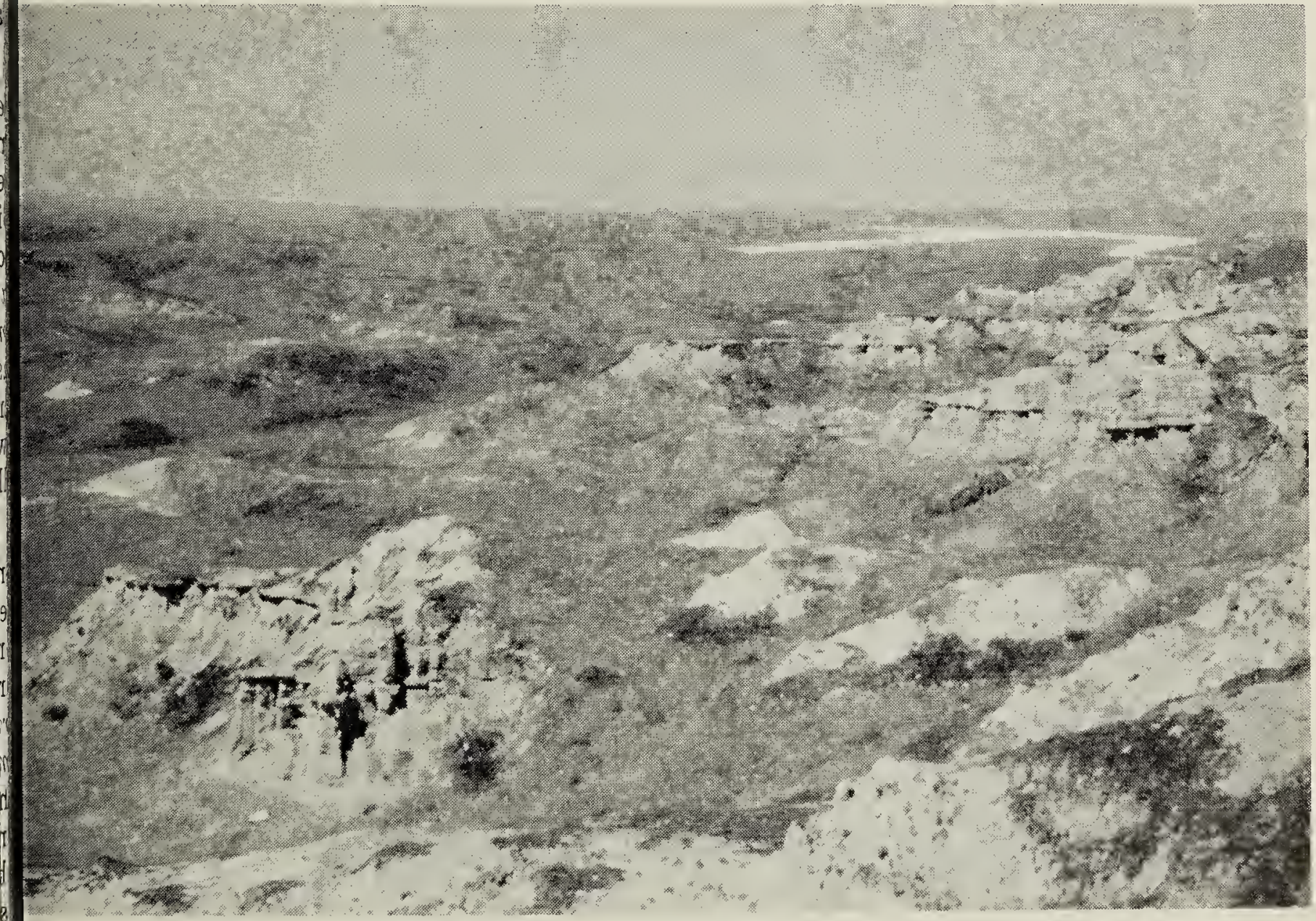

ig. 3. Badlands scuth of Big Muddy Lake (top right) showing steep nongetated slopes and less steep vegetated slopes. 


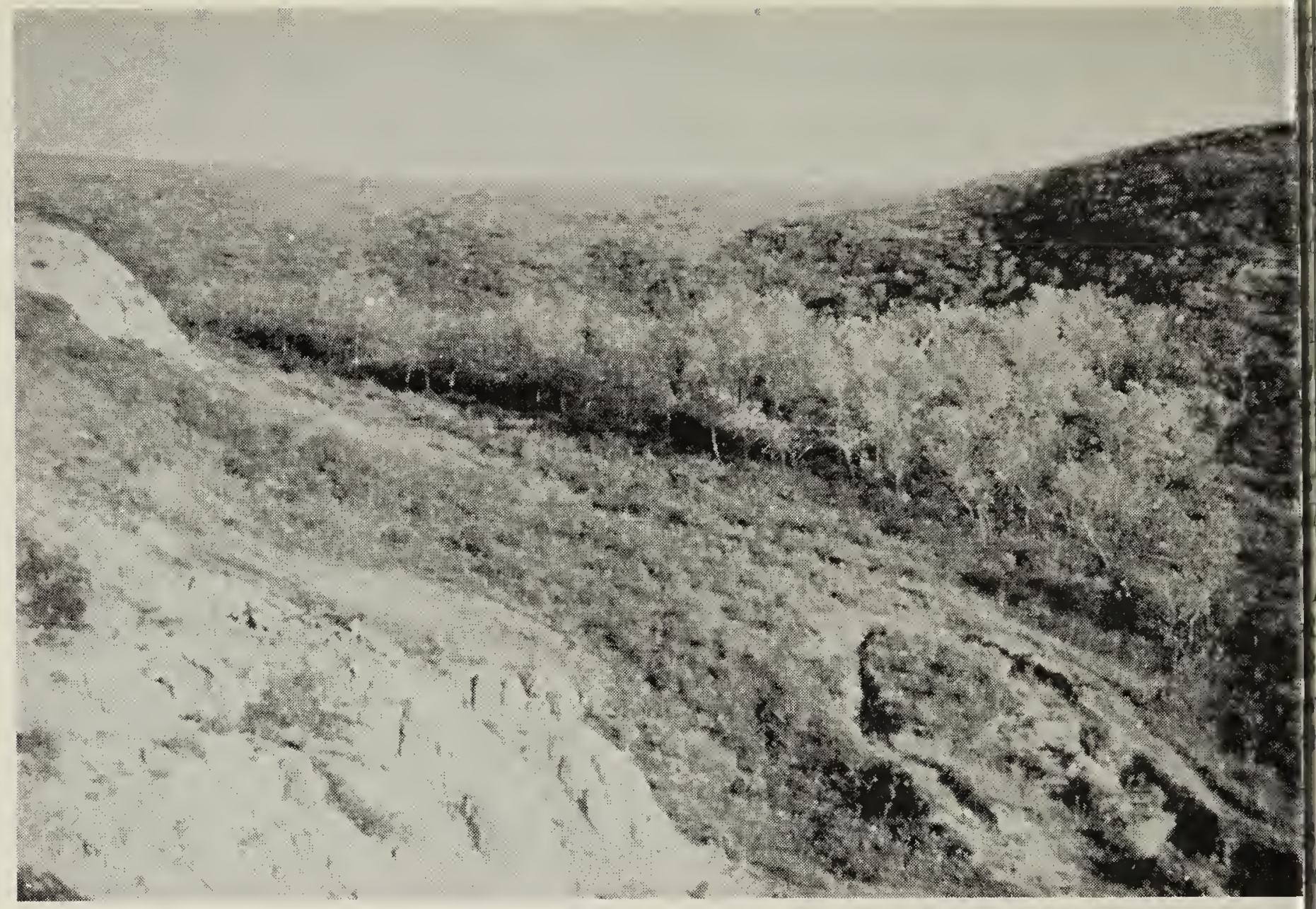

Fig. 4. Wooded depression on the northeast side of Big Coulee on the Nobl ranch. Trees are mostly Green Ash, Manitoba Maple and American Elm.

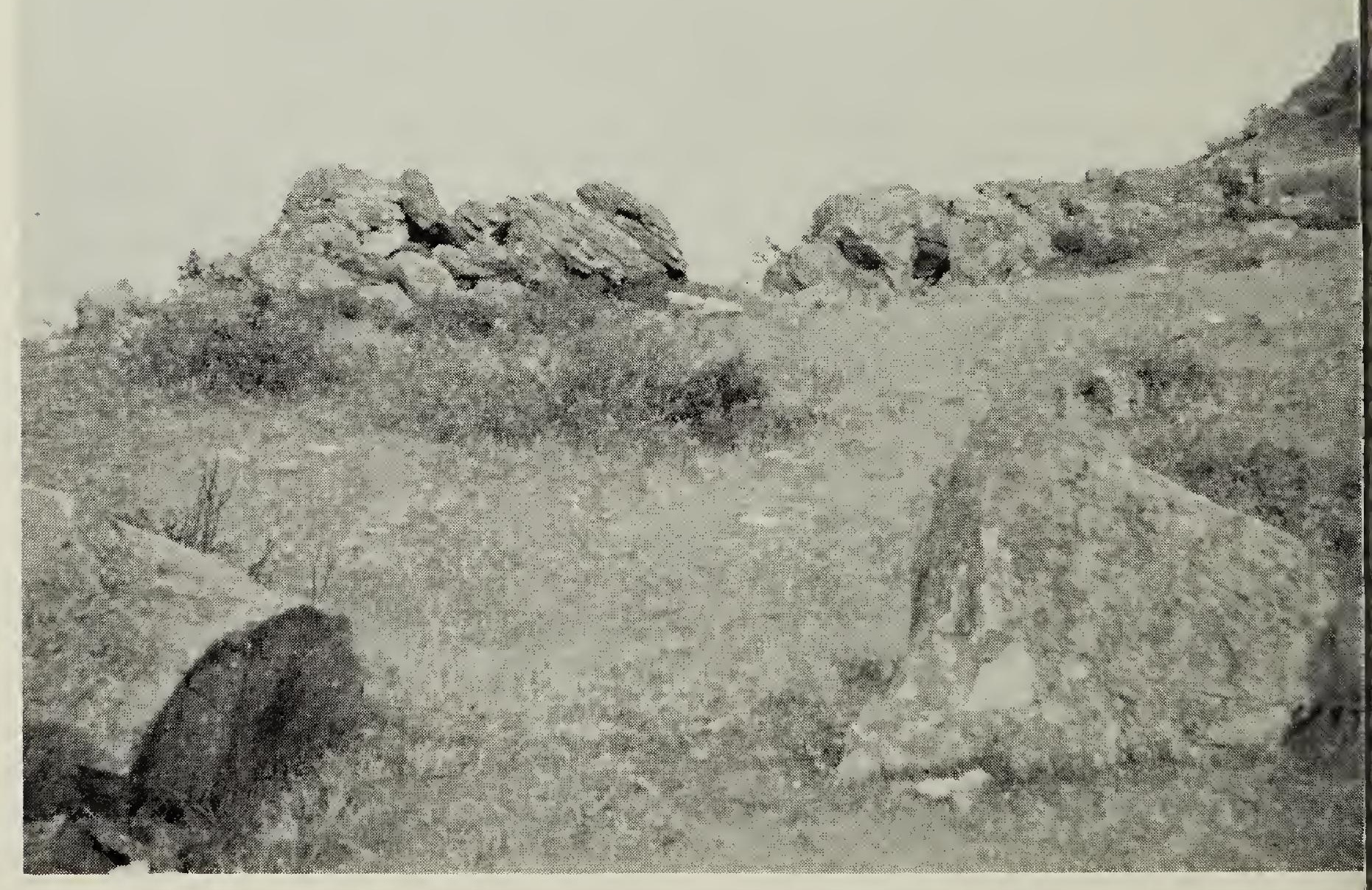

Fig. 5. Sandstone rock outcrops at Marshall ranch (site 14). 
w Umbrellaplant (Eriogonum avum), Branched Eriogonum ( $E$. ulticeps) and Spatulate Bladderpod Lesquerella alpina var. spathulata) emed to prefer dry clay banks. Evengstar (Mentzelia decapetala) was und on one occasion only.

In the "badlands", the moist depresons were filled with Aspen (Populus emuloides), American Elm (Ulmus nericana), Green Ash (Fraxinus nnsylvanica subintegerrima), under hich were Saskatoon (Amelanchier nifolia), Chokecherry (Prunus virniana var.melanocarpa), Pin Cherry Prunus pennsylvanica), and Wood's ose (Rosa woodsii), (Figure 4). uffaloberry (Shepherdia argentea) as very common in coulees and roughout the "badlands".

Three species unusual for southern askatchewan were collected from ck outcrops. Purple Cliff Brake fellaea glabella), reported south of le Missouri River in North Dakota Stevens (1950), at Lake Athaisca and in the Flin Flon area by reitung (1957), and in Manitoba by coggan (1957), was collected from indstone rock outcrops at the Marhall Ranch in the lower Big Muddy alley (Figure 5) and near West eaver Creek. This suggests that it as moved up the northern triburies of the Missouri River. It was ceviously collected in the Big Muddy B. Boivin in July, 1954 (Duplicate Fraser Herbarium). Oregon Wooda (Woodsia oregana) has been reprted in the Rocky Mountains, Cyess Hills, Drumheller, and Lake thabasca regions of Alberta by Moss 1959); Breitung (1957) reported it Cypress Hills, Denare Beach and ake Athabasca in Saskatchewan; and cevens (1950) recorded it for North akota. This was also collected on the indstone outcrop at Marshall's anch, but it was previously collected ear Bullkin Lake by B. Boivin. inear-leaved Umbrellawort (Miralis linearis) was collected in the me locality and is not commonly und north of the International oundary.
The list which follows catalogues all the species collected in the Big Muddy Valley area. The scientific names are according to Scoggan (1957). Specimens have been added to the herbarium at University of Saskatchewan, Regina Campus, and duplicates are in the Fraser Herbarium, University of Saskatchewan, Saskatoon.

\section{SPECIES LIST} EQUISET ACEAE

Equisetum arvense L., Equisetum laevigatum A. Br.

\section{SELAGINELLACEA
ella densa Rydb. \\ POLYPODIACEAE}

Cystopteris fragilis (L.) Bernh., Pellaea glabella Mett., Woodsia ore. gana D.C. Eat.

PINACEAE

Juniperus communis L., Juniperus horizontalis Moench.

Typha latifolia L.

\section{TYPHACEAE}

\section{SPARGANIACEAE}

S parganium multipedunculatum (Morong) Rydb.

\section{JUNC AGINACEAE}

Triglochin maritima L., Triglochin palustris L.

\section{ALISMATACEAE}

Alisma subcordatum Raf., Alisma triviale Pursh, Sagittaria cuneata Sheldon

\section{FESTUCEAE}

\section{GRAMINEAE}

Bromus porteri (Coult.) Nash, Bromus inermis Leyss., Distichlis stricta (Torr.) Rydb., Glyceria grandis Wats., Poa arida Vasey, Poa canbyi (Scribn.) Piper, Poa compressa L., Poa cusickii Vasey, Poa palustris L., Poa pratensis L., Poa secunda Presl, Puccinellia nuttalliana (Schult.) Hitchc.

\section{HORDEAE}

Agropyron cristatum (L.) Gaertn., Agropyron repens (L.) Beauv., Agropyron smithii Rydb., Agropyron spicatum (Pursh) Scribn. \& Smith, Agropyron trachycaulum (Link) Malte, Agropyron trachycaulum (Link) Malte var. glaucum (P. \& M.) Malte, Agropyron trachycaulum (Link) Malte var. novae-angliae (Scribn.) Fern., Agro- 
pyron trachycaulum (Link) Malte var. unilaterale (Cassidy) Malte, Elymus canadensis L., Elymus virginicus L., Hordeum jubatum L., Hordeum jubatum L. var. caespitosum (Scribn.) Hitchc.

\section{AVENEAE}

Avena fatua L., Koeleria cristata (L.) Pers.

\section{AGROSTIDEAE}

Agrostis scabra Willd., Calamagrostis inexpansa A. Gray, Calamagrostis montanensis Scribn., Calamovilfa longifolia (Hook.) Scribn., Muhlenbergia cuspidata (Nutt.) Rydb., Muhlenbergia racemosa (Michx.) BSP., Stipa comata Trin. \& Rupr., Stipa viridula Trin.

\section{CHLORIDEAE}

Beckmannia syzigachne (Steud.) Fern., Bouteloua gracilis (HBK.) Lag,. Spartina gracilis Trin.

ANDROPOGONEAE

Andropogon scoparius Michx.

\section{PANICEAE}

Echinochloa pungens (Poir.) Rydb. var wiegandii Fassett, Setaria viridis (L.) Beauv.

\section{CYPERACEAE}

Carex atherodes Spreng., Carex backii Boott, Carex brevior (Dew.) Mack., Carex stenophylla Wahl. var. enervis (Mey.) Kukenth., Carex filifolia Nutt., Carex lanuginosa Michx., Carex parryana Dew., Carex praegracilis Boott, Carex rossii Boott, Carex sprengelii Dew., Carex xerantica Bailey, Elencharis palustris (L.) R. \& S., Scirpus acutus Muhl., Scirpus americanus Pers., Scirpus nevadensis Wats., Scirpus paludosus Nels., Scirpus validus Vahl.

\section{LEMNACEAE}

Lemna minor L. (no specimen taken)

\section{JUNCACEAE}

Juncus balticus Willd. var. littoralis Engelm., Juncus bufonius L.

\section{LILIACEAE}

Allium textile Nels. \& Macbr., Disporum trachycarpum (Wats.) B.\& H., Lilium philadelphicum L. var. andi- num' (Nutt.) Ker, Smilacina stellato (L.) Desf., Smilax herbacea L. var lasioneura (Hook.) A. DC., Zigadenus gramineus Rydb.

\section{SALICACEAE}

Populus deltoides Marsh. var. occi dentalis Rydb., $\mathrm{x}$ Populus dutilly Lepage $=P$. balsamifera $\times P$. tremuloides, Populus tremuloides Michx. Salix amygdaloides Anderss., Salia bebbiana Sarg., Salix interior Rowlee Salix petiolaris Smith.

\section{ULMACEAE}

Ulmus americana $\mathrm{L}$.

\section{CANNABIN ACEAE}

Humulus lupulus L.

\section{URTICACEAE}

Parietaria pensylvanica $\mathrm{M} u \mathrm{u} l$. Urtica dioica L. var. procera Wedd (no specimen taken).

\section{SANTALACEAE}

Comandra pallida A. DC.

\section{POLYGONACEAE}

Eriogonum flavum Nutt., Eriogonum multiceps Nees, Polygonum avi. culare L., Polygonum coccineum Muhl. Polygonum convolvulus L., Polygonum la pathif olium L. var. salicifolium Sibth., Polygonum ramosissimum Michx., Rumex fennicus Murb., Rumex maritimus L. var. fueginus (Phil.) Dusen., Rumex mexicanus Meisn. Rumex occidentalis Wats.

\section{CHENOPODIACEAE}

Atriplex argentea Nutt., Atriples nuttallii Wats., Atriplex patula L. Atriplex patula L. var. hastata (L.) Gray, Axyris amaranthoides L., Chen. opodium album L., Chenopodium fre. montii Wats., Chenopodium hybridum L. var. gigantospermum (Aellen) Rouleau, Chenopodium leptophyllum Nutt. Chenopodium strictum Roth, Endolepis suckleyi Torr., Eurotia lanata (Pursh) Moq., Kochia scoparia (L.) Schrad. Sulicornia rubra Nels., Salsola kali L var. tenuifolia Tausch, Sarcobatus vermiculatus (Hook.) Torr., Suaeda depressa (Pursh) Wats.

\section{AMARANTHACEAE}

Amaranthus retroflexus $\mathrm{L}$. 
NYCTAGIN ACEAE

Mirabilis linearis (Pursh) Heimerl.

\section{CARYOPHYLLACEAE}

Cerastium arvense L., Gypsophila aniculata L., Spergularia marina L.) Griseb. var. leiosperma (Kindb.) urke, Stellaria crassifolia Ehrh., tellaria longipes Goldie.

\section{RANUNCULACEAE}

Anemone canadensis L., Anemone atens L. var. wolfgangiana (Bess.) och, Clematis ligusticifolia Nutt., anunculus cymbalaria Pursh, Ranuilus glaberrimus Hook. var. ellipticus reene, Ranunculus macounii Britt., anunculus sceleratus L., Ranunculus rcinatus Sibth. var. subrigidus Drew) Benson.

\section{PAPAVERACEAE}

Corydalis aurea Willd.

\section{CAPPARID ACEAE}

Cleome serrulata Pursh.

\section{CRUCIFERAE}

Arabis divaricarpa, Nels., Arabis lboellii Hornem. var. retrofracta Graham) Rydb., Armoracia lapathilia Gilib., Brassica kaber (DC) L. Wheeler, Capsella bursa-pastoris .) Medic., Conringia orientalis (L.) umort., Descurainia pinnata (Walt.) ritt. var. brachycarpa (Richards.) ern., Descurainia richardsonii Sweet) O. E. Schulz, Descurainia phia (L.) Webb, Draba memorosa Erysimum asperum (Nutt.) DC., ysimum cheiranthoides L., Erysium conspicuum (Wats.) MacM., Hesris matronalis L., Lepidium densiorum Schrad., Lesquerella alpina Nutt.) Wats.var.spathulata (Rydb.) ayson, Lesquerella ludoviciana Nutt.) Wats., Sisymbrium altissium L., Thlaspi arvense L.

\section{SAXIFRAGACEAE}

Ribes americanum Mill., Ribes ireum Pursh, Ribes oxyacanthoides

\section{ROSACEAE}

Amelanchier alnifolia Nutt., Chaaerhodos erecta (L.) Bunge ssp. ittallii (Pickering) Hult., Crataegus rotundifolia Moench, Geum aleppicum Jacq. var. strictum (Ait.) Fern, Geum triflorum Pursh, Potentilla anserina L., Potentilla arguta Pursh, Potentilla concinna Richardson, Potentilla fruiticosa L., Potentilla gracilis Dougl., Potentilla pensylvanica L., Potentilla pensylvanica var. bipinnatifida (Dougl.) T.\& G., Potentilla plattensis Nutt., Prunus pensylvanica L.f., Prunus virginiana L. var. melanocarpa (Nels.) Sarg., Rosa arkansana Porter, Rosa woodsii Lindl.

\section{LEGUMINOSAE}

Astragalus bisulcatus (Hook.) Gray, Astragalus canadensis L., Astragalus flexuosus Dougl., Astragalus goniatus Nutt., Astragalus pectinatus Dougl., Astragalus striatus Nutt., Astragalus tenellus Pursh, Astragalus triphyllus Pursh, Caragana arborescens Lam., Glycyrrhiza lepidota (Nutt.) Pursh, Lotus purshianus (Benth.) Clements \& Clements, Medicago falcata L., Medicago sativa L., Melilotus alba Desr., Melilotus officinalis (L.) Lam., Oxytropis sericea Nutt. var. spicata (Hook.) Barneby, Petalostemum candidum (Willd.) Michx., Petalostemum purpureum (Vent.) Rydb., Psoralea argophylla Pursh, Psoralea esculenta Pursh, Thermopsis rhombifolia (Nutt.) Richards., Vicia americana var. angustifolia Nees.

\section{LIN ACEAE}

Linum lewisii Pursh, Linum rigidum Pursh.

\section{POLYGALACEAE}

Polygala alba Nutt.

\section{EUPHORBI ACEAE}

Euphorbia glyptosperma Engelm.

\section{ANACARDIACEAE}

Rhus radicans L. var. rydbergii (Small) Rehd. (no specimen taken), Rhus trilobata Nutt.

\section{ACERACEAE}

Accr negundo L.

\section{MALVACEAE}

Sphaeralcea coccinea (Pursh) Rydb.

\section{VIOLACEAE}

Viola adunca Sm., Viola nuttallii Pursh, Viola rugilosa Greene. 
LOASA CEAE

Mentzelia decapetala (Pursh) Urban \& Gilg.

\section{CACT ACEAE}

Mamillaria vivipara (Nutt.) Haw., Opuntia fragilis (Nutt.) Haw., Opuntia polyacantha Haw.

\section{ELAEAGNACEAE}

Elaeagnus commutata Bernh., Shepherdia argentea Nutt., Shepherdia canadensis (L.) Nutt.

\section{ONAGRACEAE}

Epilobium glandulosum Lehm., Epilobium leptophyllum Raf., Gaura coccinea Pursh, Oenothera biennis L. var. canescens Torr. \& Gray, Oenothera caespitosa Nutt.

\section{HALORAGACEAE}

Myriophyllum exalbescens Fern.

\section{HIPPURIDACEAE}

Hippuris vulgaris L.

\section{UMBELLIFERAE}

Cicuta douglasii (DC.) Coult. \& Rose, Lomatium foeniculaceum (Nutt.) Coult. \& Rose, Musineon divaricatum (Pursh) Nutt., Sanicula marilandica L., Sium suave Walt., Zizia aptera (Gray) Fern.

\section{CORNACEAE}

Cornus stolonifera Michx.

\section{PYROLACEAE}

Pyrola secunda L.

\section{PRIMULACEAE}

Androsace occidentalis Pursh, Androsace septentrionalis L., Dodecatheon radicatum Greene, Steironema ciliatum (L.) Raf.

\section{OLEACEAE}

Fraxinus pennsylvanica Marsh. var. subintegerrima (Vahl) Fern.

\section{GENTIANACEAE}

Gentiana affinis Griseb., Gentiana amarella L.

\section{ASCLEPIADACEAE}

Asclepias speciosa Torr.

\section{CONVOLVULACEAE}

Convolvulus arvensis $\mathrm{L}$.

\section{POLEMONIACEAE}

Collomia linearis Nutt., Phlox alyssifolia Greene, Phlox hoodii Richards.

\section{BORAGINACEAE}

Cryptantha bradburiana Pays Hackelia americana (Gray) Fer Heliotropium curassavicum L. v obovatum DC., Lappula echinata Gil Lappula redowsliii (Hornem.) Gree var. occidentalis (Wats.) Rydb., Lit spermum incisum Lehm., Merten lanceolata (Pursh) DC.

\section{LABI AT AE}

Agastache foeniculum ( P u r s Ktze., Lycopus asper Greene, Ment arvensis L. var. villosa (Bent Stewart, Monarda fistulosa L., S chys palustris L. var. pilosa (Nut Fern.

\section{SOLAN ACEAE}

Solanum triflorum Nutt.

\section{SCROPHULARIACEAE}

Castilleja sessiliflora Pursh, Orth carpus luteus Nutt., Penstemon al dus Nutt., Penstemon nitidus Doug

\section{PLANTAGINACEAE}

Plantago elongata Pursh, Planta eriopoda Torr., Plantago major Plantago purshii R. \& S.

\section{RUBIACEAE}

Galium septentrionale R.\& S.

\section{CAPRIFOLIACEAE}

Symphoricarpos albus (L.) Blal Symphoricarpos occidentalis Hook.

CAMPANULACEAE

Campanula rotundifolia $\mathrm{L}$.

\section{COMPOSITAE}

Achillea lanulosa Nutt., Agoser cuspidata ( Pursh) Raf., Agoser glauca (Nutt.) Greene, Ambrosia art misiifolia L. var. elatior (L.) Descou tils, Antennaria aprica Greene, Ante naria neglecta Greene, Antennar parvifolia Nutt., Arctium min (Hill) Bernh., Artemisia cana Purs Artemisia caudata Michx. var. calve Lunell, Artemisia frigida Willd., Art misia glauca Pall., Artemisia long folia Nutt., Artemisia ludovicia Nutt. var. gnaphalodes (Nutt.) T. G., Aster brachyactis Blake, Ast canescens Pursh, Aster chilensis $\mathrm{Ne}$ ssp. adscendens (Lindl.) Cronq., Ast 
lcatus Lindl., Aster hesperius Gray, ster laevis L. var. geyeri Gray, Aster insus (Blake) Cronq., Aster pauciorus Nutt., Bidens cernua L., Chrypsis villosa (Pursh) Nutt., Chrysoamnus nauseosus (Pall.) Britt., Cirum arvense (L.) Scop., Cirsium odmanii (Rydb.) Arthur, Cirsium idulatum (Nutt.) Spreng., Crepis incinata (James) T. \& G., Crepis incinata (James) T. \& G. subsp. auca (Nutt.) Babcock \& Stebbins, chinacea angustifolia DC., Erigeron per Nutt., Erigeron caespitosus utt., Gaillardia aristata Pursh, Grin-lia squarrosa (Pursh) Dunal var. casiperennis Lunell, Gutierrezia saro. rae (Pursh) Britt. \& Rusby, Haplotppus lanceolatus (Hook.) T. \& G., aplopappus spinulosus (Pursh) DC. elianthus annuus L., Helianthus etiflorus Pers. var. subrhomboideus iydb.) Fern., Helianthus nuttallii $\mathrm{T}$. G., Hymenopappus filifolius Hook., ymenoxys richardsonii (Hook.) Cock-ell, Iva axillaris Pursh, Iva xanthilia Nutt., Lactuca pulchella (Pursh) C., Lactuca scariola L., Liatris ligutylis (Nels.) K. Schum., Liatris nctate Hook., Lygodesmia juncea ursh) D. Don., Ratibida columnira (Nutt.) Woot. \& Standl., Senecio nus Hook., Senecio integerrimus utt., Solidago canadensis L. var. lvocanescens Rydb., Solidago gigana Ait. var. serotina (Kuntze) Cronq., lidago missouriensis Nutt., Solidago ollis Bartl., Solidago rigida L., Sonus arvensis L., Sonchus arvensis L. ar. glabrescens Guenth., Grab., imm., Sonchus asper (L.) Hill, anacetum vulgare L., Taraxacum ythrospermum Andrz., Taraxacum ficinale Weber, Tragopogon dubius cop., Townsendia exscapa (Richds.) Porter, Xanthium strumarium

\section{ummary}

This paper lists 336 species of vaslar plants in 61 different families und in 1968 in the Big Muddy gion. Some species were common id abundant while others appeared be rare but in this preliminary study no attempt was made to evaluate relative abundance. It is hoped that much more information about the plants of this interesting area will be obtained. Voucher specimens for this situdy have been placed in the University herbaria in both Saskatoon and Regina. The collection in Regina will be kept as a separate unit for several years so that interested botanists can examine species reported in this paper and so that additions to the flora of the area may be easily made.

\section{Acknowledgments}

I wish to express my appreciation to Dr. G. F. Ledingham of the University of Saskatchewan, Regina Campus, for the encouragement and guidance he gave me in carrying out this project and for his assistance in the field and laboratory. I gratefully acknowledge the aid of Mr. J. H. Hudson of the Saskatchewan Research Council Engineering Division, Saskatoon, who identified some of the collections and who verified all of the specimens collected. I wish, also, to thank Dr. G. W. Argus of the University of Saskatchewan, Saskatoon Campus, for his help in the identification of Salix species. To Mr. Richard Fyfe of Canadian Wildlife Service, Mr. Bernie Haysom, and those friends who assisted me in the field, I express my thanks. I especially thank Mr. and Mrs. W. Noble for their warm hospitality during my visits to the Big Muddy.

This project was supported by the National Research Council of Canada.

\section{LITERATURE CITED}

Breitung, A. J. 1957. Annotated catalogue of the vascular flora of Saskatchewan. Amer. Midl. Natur. 58(1):1-72.

Houldsworth, E. 1941. The Big Muddy Valley of southern Saskatchewan. Can. Geogr. J. $23(3): 116-131$.

Moss, E. H. 1959. Flora of Alberta. University of Toronto Press, Toronto. $546 \mathrm{p}$.

Parizek, R. R. 1964. Geology of the Willow Bunch Lake area (72-H) Saskatchewan. Sask. Res. Council Geol. Div. Rep. No. 4.

Scoggan, H. J. 1957. Flora of Manitoba. Nat. Mus. of Can. Bull. No. 140. 619 p.

Stevens, O. A. 1950. Handbook of North Dakota plants. N.D. Inst. for Reg. Stud., Fargo. 324 p. 